Article

\title{
Some Topics on the Physics of Bubble Dynamics in Beer
}

\author{
Patricia Vega-Martínez, Oscar R. Enríquez and Javier Rodríguez-Rodríguez * \\ Fluid Mechanics Group, Carlos III University of Madrid, 28911 Leganés (Madrid), Spain; \\ pvega@ing.uc3m.es (P.V.-M.); oenrique@ing.uc3m.es (O.R.E.) \\ * Correspondence: javier.rodriguez@uc3m.es \\ Academic Editors: Edgar Chambers IV and Ombretta Marconi \\ Received: 15 May 2017; Accepted: 14 July 2017; Published: 27 July 2017
}

\begin{abstract}
Besides being the favorite beverage of a large percentage of the population, a glass or bottle of beer is a test bench for a myriad of phenomena involving mass transfer, bubble-laden flows, natural convection, and many more topics of interest in Physical Chemistry. This paper summarizes some representative physical problems related to bubbles that occur in beer containers, pointing out their practical importance for the industry of beverage processing, as well as their potential connection to other processes occurring in natural sciences. More specifically, this paper describes the physics behind the sudden foam explosion occurring after a beer bottled is tapped on its mouth, gushing, buoyancy-induced motions in beer glasses, and bubble growth in stout beers.
\end{abstract}

Keywords: bubbles; henry's law; convective phenomena; mass transport; diffusion

\section{Introduction}

Every beer lover enjoys the taste and texture of a well-served beer. However, if the drinker is also a person with some scientific curiosity or interest beyond the physical satisfaction that this beverage induces, surely she or he will be able to appreciate a different kind of pleasure. Namely, the observation of a rich variety of physical phenomena that develop around a beer glass or bottle. To name a few, the sudden generation of foam when the container is shaken or hit, the development of jets and plumes of bubbles that rise to the surface, the formation of a foam head when the beer is properly drafted, or even the appearance of foamy waves that travel downwards near the glass wall, defying the common intuition that bubbles rise, not sink. All these phenomena-and many others-have in common that they are driven by the interplay between bubble growth in supersaturated liquids, buoyancy, and the transport of gases dissolved in a liquid in motion (what is called advection). In this paper, we have picked three specific physical problems as vehicles to discuss the Physics behind these effects in a qualitative yet rigorous way.

Thus, the goal of the paper is not to give a precise quantitative or mathematical description of bubble-laden processes occurring in beer, but rather to lure readers from different backgrounds (but with a common liking for beer) into enjoying the physics of bubbles in beer. Besides the articles that we cite in the following sections, those readers interested in digging deeper into the mathematical models are referred to the excellent review of Liger-Belair [1] on the similar topic of bubble physics in Champagne (a beverage with which beer has a great deal in common from the physical point of view).

For the sake of conciseness, the present article does not cover other natural phenomena related to beer, such as the biochemistry of fermentation or thermodynamic phenomena associated with beer production and transport. These and other topics are beautifully described in the books by Mark Denny [2] and Charles Bamforth [3]. 
Before starting with our excursion through the physics of bubbles in beer, we will devote the end of this section to the introduction of some essential concepts that the reader will need in order to understand what is explained next.

\subsection{A Brief Scientific Description of Carbonated Beverages}

The two essential ingredients of bubbly drinks are water and carbon dioxide. Of course, their flavor and texture become more sophisticated when incorporating other ingredients, which usually come from plants (fruits, cereals, flowers, roots, etc.), but sometimes from chemistry labs. The carbon dioxide they contain might be generated within through fermentation, or may be injected and dissolved through mechanical means. In either case, the inside of a closed container with such a beverage is at a pressure higher than atmospheric. It is at the moment of opening (or pouring through a tap) that its bubbly bountifulness is released. We now explain some physical principles that are at the heart of bubble growth and motion in drinks, and that are necessary to discuss the rest of the topics in this piece.

\section{Henry's Law and Oversaturation}

The first principle-Henry's law-states that the amount of a gas that can be dissolved (its equilibrium concentration) in a liquid is proportional to the partial pressure of that gas above the liquid; this is

$$
c=k_{H} P
$$

The concentration $c$ is the amount of solute (in mass, volume, or moles) per unit volume of the mixture. The proportionality constant $k_{H}$ is known as Henry's coefficient or constant; it is specific to each gas-liquid pair, and decreases with increasing temperature. One must always be careful with units and temperature dependence when using Henry's coefficient [4].

Hence, considering the minute partial pressure of $\mathrm{CO}_{2}$ in the atmosphere, one can understand why carbonated drinks need to be held under pressure in order to contain this dissolved gas. As long as they are pressurized, the gas concentration is at equilibrium, but upon opening their pressure drops to atmospheric level and the liquid suddenly finds itself containing much more dissolved gas than it "wants" to. This situation-known as oversaturation-is thermodinamically unstable, and therefore gas must exit the solution through its free surface until equilibrium is regained. How it exits is at the heart of the phenomena that we will discuss further. As a note, pressure in closed beverages goes from 2 to 6 times atmospheric pressure (around 2-5 for beer and 6 for Champagne wines). An oversaturation coefficient $\zeta$ can be defined as a relation between the bottling pressure $P_{b}$ and atmospheric pressure $P_{\text {atm }}$ as:

$$
\zeta=\frac{P_{b}-P_{\mathrm{atm}}}{P_{\mathrm{atm}}}
$$

With the bottling pressures mentioned above, we expect $\zeta \approx 1-4$ for beer and $\zeta \approx 5$ for Champagne.

\section{Bubble Nucleation and Growth}

Once a drink is oversaturated, a crucial question is how bubbles come to form. This implies creating a gas-liquid interface within the liquid matrix, which has an energetic cost. The interested reader can find an account of the different types of bubble nucleation in the review by Jones, Evans, and Galvin [5]. At the oversaturation conditions of beverages, bubble formation requires the presence of nucleation sites, which might be provided by contaminating particles (such fabric fibers left in a glass) or crevices in the container. 
The energy implied in the existence of an interface (i.e., surface tension, $\sigma$ ), gives rise to an overpressure $\Delta P_{L}$ known as Laplace Pressure [6] at the inside of the bubble (of radius $R$ ) given by

$$
\Delta P_{L}=\frac{2 \sigma}{R}
$$

In equilibrium conditions, this overpressure will lead to the dissolution of the bubble, whereas in an oversaturated liquid it will determine the critical (minimum) radius $R_{c}$ that a bubble needs to have in order to grow:

$$
R_{c}=\frac{2 \sigma}{P_{b}-P_{\mathrm{atm}}} .
$$

For example, in a beer bottled at $P_{b} \approx 3.5 P_{\text {atm }}$, with a surface tension $\sigma=0.04 \mathrm{~N} / \mathrm{m}$ [7], the critical radius is $R_{c} \sim 0.3 \mu \mathrm{m}$. This size is indeed much smaller that the typical bubble nuclei found in beer. For instance, cellulose fibers-which serve as nucleation sites when present-have typical diameters between 10 and $20 \mu \mathrm{m}$.

Bubbles then grow (or dissolve) due to the diffusion of $\mathrm{CO}_{2}$. The essence of this process was explained by Epstein and Plesset [8] considering the ideal situation of an isolated bubble which is at rest. The key assumption is that the gas concentration $c_{S}$ at the bubble surface (on the liquid side) is in a Henry equilibrium state. For the case of a bubble in an open beverage, this is $c_{s}=k_{H}\left(P_{\mathrm{atm}}+\Delta P_{L}\right)$, and if this is smaller than the concentration at bottling, $c_{b}=k_{H} P_{b}$ diffusion takes place towards the bubble. Although the situation of bubbles in a beverage is far from this theoretical scenario-there might be many neighboring bubbles which are also moving-the bubble radius still grows proportionally to the square root of time, as explained by Epstein and Plesset.

\section{Buoyancy and Circulating Flows}

Finally, the fact that bubbles rise will be of paramount importance in the following discussions. Their ascent is due to buoyancy $F_{b}$, described by Archimedes as the upwards force experienced by any body submerged in a liquid. This force is equal to the weight of the displaced liquid volume. For the case of a sphere, $F_{b}=(4 / 3) \pi R^{3} g \rho_{\text {liq }}$, with $g$ being gravitational acceleration. Whether an object rises, sinks, or floats neutrally depends on the balance between its weight and buoyancy force; in the case of a bubble, since its density $\rho_{\text {gas }}$ is much smaller than the liquid's, upwards buoyant force will always be larger than its weight $W=(4 / 3) \pi R^{3} g \rho_{\text {gas }}$.

An ascending bubble displaces the fluid along its path, creating a circulating motion of the liquid around it. The collective action of several bubbles rising at different velocities from various locations creates a mixing effect which affects taste and composition of the beverage, leading, for example, to a faster degassification than would occur solely by diffusion [9-12]. This mixing effect of bubbles is also used in bio reactors [13].

\section{Explosive Foam Generation in Beer Tapping}

Perhaps of all the phenomena occurring in a beer (and also in Champagne, see Video S1), the one that involves more physical effects is the so-called "beer tapping". It consists in the explosive generation of foam after a (usually freshly opened) beer bottle is hit on its mouth. Almost everyone has the experience of either having suffered this prank in a bar or having made someone suffer with it.

Although it is easy to infer that the impact triggers cavitation and that the formation of foam is a consequence of this, two features of the phenomenon clearly suggest that cavitation alone is unable to explain the observations. Namely,

1. Cavitation occurs only at a handful of locations, whereas the foam eventually fills the whole bottle.

2. Cavitation is very fast, taking place in a matter of a fraction of a millisecond, much less than the time -around a second- that it takes for the foam to spill. 
Therefore, two questions are left open if only cavitation is considered: what makes cavitation bubbles grow and/or multiply to fill the whole container? Additionally, what happens between the inception of cavitation —nearly simultaneous to the impact-and the spilling of the foam?

Rodríguez-Rodríguez, Casado-Chacón, and Fuster [14] performed an experimental study of the succession of processes that occur in a beer bottle after an impact, using high-speed imaging and pressure-time signals from a hydrophone. In this way, they were able to reconstruct the chain of phenomena that lead to the generation of foam. In summary, the different stages that take place in the bottle are these:

Wave propagation-cavitation stage: the impact on the mouth of the bottle creates a compression wave that runs downward along the glass. Since the bottom of the bottle is a free-end boundary condition (i.e., no pressure is applied there), when this wave reaches the bottom of the bottle, it bounces back along the glass and liquid as an expansion wave. This wave provokes a sudden pressure drop in the liquid and thus the formation of cavitation bubbles. A different explanation of this stage was suggested by Mantic-Lugo et al. [15]. Based on the analysis of high-speed movies of cavitation bubbles, they concluded that the pressure does not propagate as a wave inside the liquid, but that it evolves uniformly inside instead. In other words, that the acceleration of the container is the responsible for the sudden pressure drop that triggers cavitation. This conclusion is compatible with their measurements, although it does not explains the occurrence of cavitation in the experiments reported in [14], where the bottle is kept fixed during the impact. Moreover, in this same reference, it can be observed how bubbles start to grow at different times, first near the bottom and then close to the free surface, in opposition to the uniform-pressure hypothesis. It should be pointed out that the mechanism that triggers cavitation has no effect on the subsequent evolution of the bubbles. Indeed, beer tapping can even be induced without any mechanical impact by focusing a laser pulse inside a bottle [14].

Being the acceleration of the container or a travelling acoustic wave that is responsible for the inception of cavitation, it seems quite clear that an oscillatory pressure inside the bottle is essential to induce bubble collapse and thus the formation of very small bubble fragments. This occurs in times on the order of one tenth of a millisecond.

Diffusion-driven stage: upon bubble collapse, the formation of a large number of bubble fragments suddenly increases the gas-liquid contact area. This has the effect of enhancing mass transfer and thus bubble growth. It should be pointed out that in order for this to occur, these fragments must be large enough so that capillary, or Laplace, pressure does not lead to bubble dissolution. Some approximate calculations reported in [14] suggest that in beer tapping the size of the bubble fragments is around 10 microns, much larger than the typical scale at which capillary pressure dominates, which is 1 micron for bubbles at near-ambient pressures.

Because bubble growth is driven by the diffusion of dissolved $\mathrm{CO}_{2}$, the rate at which bubble sizes increase is proportional to the square root of the time, as predicted by the classical theory of Epstein and Plesset [8].

This stage covers the range between $1 \mathrm{~ms}$ and approximately $100 \mathrm{~ms}$, or a tenth of a second.

Buoyancy-driven stage: at approximately $100 \mathrm{~ms}$, buoyancy induces in the foam a velocity that is fast enough to further enhance mass transfer through advection. In other words, through the relative motion between bubble clouds and the liquid that contains fresh $\mathrm{CO}_{2}$ through which they rise. This has an explosive effect in bubble growth, and indeed it is during this stage that most of the foam volume is generated [14]. Although the exact rate at which foam volume multiplies cannot be measured due to the opacity of the bubbly flow, measurements show that these bubble plumes rise at a nearly constant speed. This is in contrast with plumes generated by a fluid parcel of constant buoyancy (such an explosion, for instance), whose velocity decays as the square root of the time, or even with radiogenic plumes like those found in the Earth's mantle, where buoyancy is generated by radioactive decay at a constant rate [16]. Thus, it seems that the degassing mechanism induced by convection in these 
bubble plumes is able to exolve gas at an accelerating pace. We must point out that the increment in the volume of the foam is purely due to bubble growth and not to the appearance of new bubbles.

The large efficiency of this mechanism in making bubbles grow is further supported by experiments in microgravity, where bubble clouds continue to grow purely by diffusion up to times on the order of several seconds [17] (see Video S4 in Supplemental Materials). Clearly, these observations reinforce the important role that buoyancy has in mass transfer in carbonated beverages-a topic that will be revisited in Section 4 .

The just-described evolution of foam formation in beer tapping is summarized graphically in Figure 1 . The four panels correspond to different time instants belonging to the three stages described above (see Figure 1).

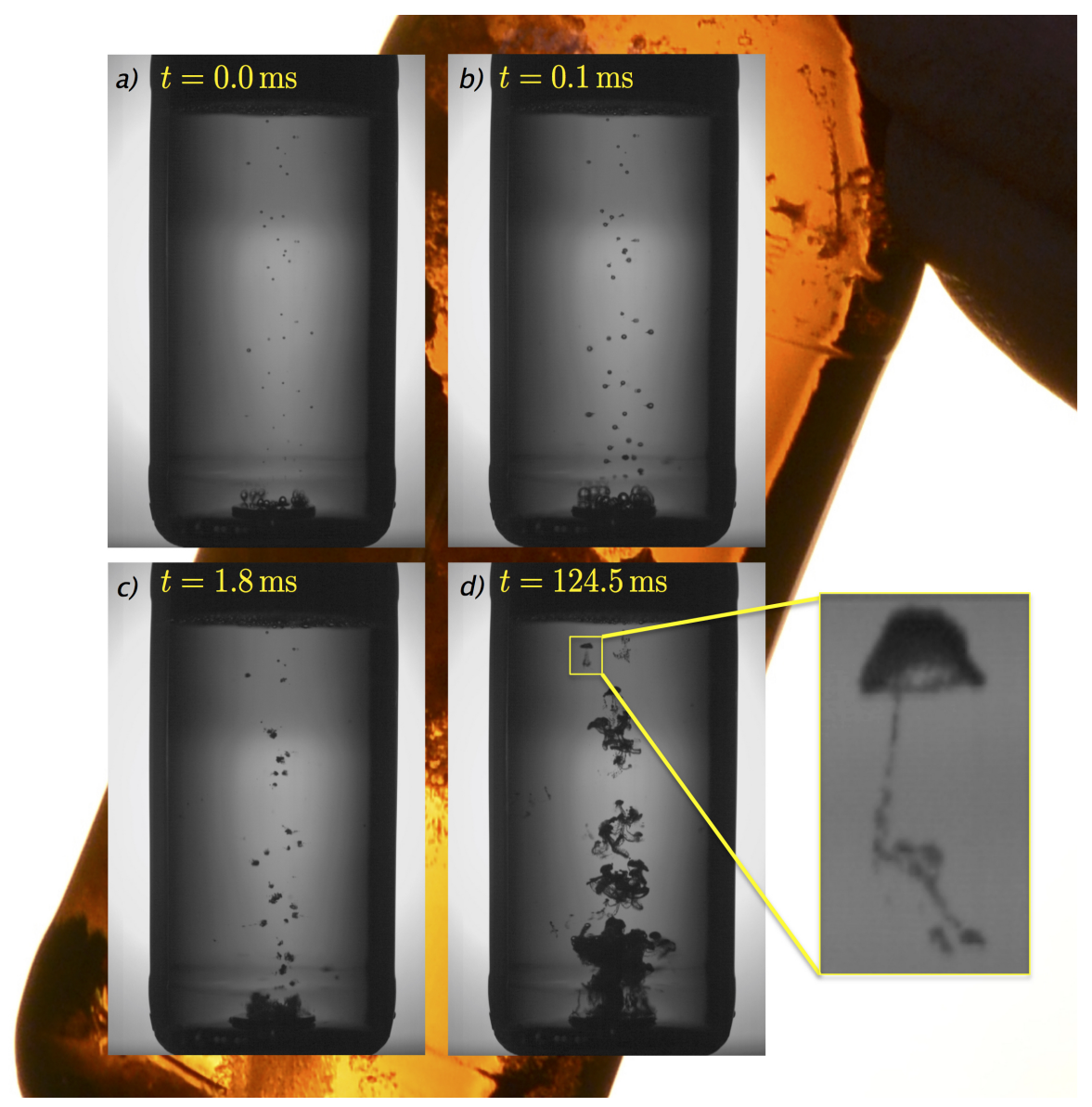

Figure 1. Different stages in the evolution of foam in the beer tapping prank. (a) $t=0 \mathrm{~ms}$, right before the impact. Notice the presence of a metal disk at the bottom to promote the formation of a bubble column. These bubbles will act as "tracers" of the processes occurring in the bottle. (b) $t=0.1 \mathrm{ms,}$ bubbles grow due to the sudden pressure drop and then quickly collapse, giving birth to tiny foam balls. (c) At $t=1.8 \mathrm{~ms}$, these foam or bubble balls grow purely due to the diffusion of $\mathrm{CO}_{2}$ into them. (d) At $t=124.5 \mathrm{~ms}$, the flow is dominated by buoyancy-driven bubbly plumes like the one shown in the inset. The relative motion between these plumes and the liquid greatly enhances mass transfer, and thus most of the foam is generated in this stage.

To conclude this section, we point out that the mechanism of explosive degassing observed in beer tapping is analogous to that occurring in limnic eruptions. This kind of eruption takes place in lakes 
whose bottom is saturated with $\mathrm{CO}_{2}$. Since water density increases with the amount of $\mathrm{CO}_{2}$ dissolved ${ }^{1}$, having a bottom saturated with this gas yields a stable stratification, and this leads to the accumulation of this gas-generated by nearby geological activity-over the years. However, for reasons not yet fully understood, a parcel of $\mathrm{CO}_{2}$-rich liquid from the bottom may rise to a depth where it becomes supersaturated, which triggers the formation of bubbles. Once these bubbles appear, buoyancy makes them rise and form a bubbly plume that keeps draining fluid from the bottom to the surface until the lake is almost degassed. The most dramatic example of this kind of eruptions took place in lake Nyos, Cameroon, in 1986 and caused about 1700 casualties by asphyxiation [18]. This flow is similar to beer tapping, in that the plume that causes the fast degassing feeds on the buoyancy that it produces and thus becomes a self-sustaining flow, which explains its explosive nature. This kind of phenomenon is also observed in chemical reactors and is known as autocatalytic plume [19]. The difference between limnic eruptions and beer tapping resides in the different mechanism that triggers the initial formation of the bubbles. It should be pointed out that although the exact cause of the 1986 accident in lake Nyos is still a matter of debate, a landslide emerges as the most likely candidate. Indeed, [20] carried out a very ingenious laboratory experiment in which they triggered a similar degassing plume by creating a "landslide" of salt grains in a tank that had a stable stratification consisting of a soda layer near the bottom and a bulk of degassed water.

\section{Effervescence and Beer Gushing}

Now we take a look at two other examples of carbonated beverage overflow, which are also due to the simultaneous growth and rise of a large number of bubbles. First, the spillage that occurs when one opens a carbonated drink after having shaken it vigorously. This type of gushing-hereafter called effervescence-can affect every type of carbonated drink. To discuss it, we will use the example of plain carbonated water, since it contains no other chemical species (such as proteins) that complicate things by introducing other physical effects (like changes in interfacial tension). Secondly, we address beer gushing-a phenomenon of significant concern for brewers which also happens right after opening a bottle, but is not necessarily associated with previous shaking. Instead, it results from the use of mold-infected malt (primary gushing) or faults during processing and handling (secondary gushing) [3].

\subsection{Effervescence}

The effects of shaking a closed carbonated drink can be illustrated with a very simple tabletop experiment. We now invite the reader to make a pause and get ahold of a transparent bottled drink (not beer yet), shake it energetically for a few seconds (say 20), place it on a surface at rest and observe what happens next. One can observe a great amount of bubbles in the liquid ascending due to buoyancy (with a speed proportional to their radius squared). Depending on the height of the bottle it will take approximately 1-3 min for visible bubbles to rise up to the head space.

While rising, bubbles are also deflating; however, this phenomenon is harder to detect by eyesight. This shrinking is due to their larger internal pressure caused by interfacial tension $(\sigma)$, and to the fact that the gas concentration in the liquid is (nearly) at Henry's equilibrium. In the end, the time it takes for bubbles to become extinct in the liquid bulk will be given by the combination of rising and shrinking rates, as analysed by Vreme et al. [21]. After this time (a few minutes), one can safely open the bottle without getting wet.

Contrarily to what intuition might suggest, Vreme et al. [21] have shown that the pressure in the bottle is not increased by the shaking. Hence, the sudden effervescence and overflow is due exclusively to the rapid simultaneous growth of a very large number of bubbles, triggered by the oversaturation

1 For instance, water saturated with $\mathrm{CO}_{2}$ at ambient pressure has a density $0.03 \%$ higher than pure water. 
established when opening the bottle. In contrast with the case of beer tapping, where the bubbles start growing from a few localized cavitation sites, here they grow everywhere in the liquid bulk.

For a given oversaturation coefficient, there is a critical size above which bubbles will grow. As an example, consider a bottle of carbonated water with $2.5 \mathrm{~atm}$ of absolute pressure in the head space. The minimum radius for a bubble to grow after a pressure drop to $1 \mathrm{~atm}$ is given by $R_{c}=2 \sigma / \Delta P_{0}$ [22]. With $\sigma=0.072 \mathrm{~N} / \mathrm{m}^{2}$ and $\Delta P_{0} \approx 1.5 \times 10^{5} \mathrm{~Pa}, R_{c} \sim 1 \mu \mathrm{m}$; hence, the vast majority of bubbles present after shaking will be larger than this size.

Going back to our experiment, it is worth noting that even long after having shaken it, depending on the bottle material, there might be a fair amount of bubbles on the wall (specially if it is plastic). In principle, there is no size for which bubbles are stable in a solution at equilibrium. However, a surface that favours bubble pinning (hydrophobic ones work best) or the presence of hydrophobic molecules that might form a shell around a bubble can stabilize bubbles for very long times, which brings us to the next type of beverage overflow discussed.

\subsection{Beer Gushing}

A nightmare that haunts brewers every now and then-gushing-is an over-foaming that occurs when opening a beer. In this case, although the physical mechanism is also the simultaneous growth and ascent of bubbles, the cause is not shaking or impact, but rather the use of mold-infected malt (known as primary gushing) or the presence of colloidal or solid particles (secondary gushing) [3].

It might be illustrative at this point to perform the same experiment we just did before, but with a reasonably translucent beer bottle. Vigorous shaking will once more produce a great number of bubbles that will also rise and disappear after a short time. However, instead of simply bursting at the surface, they will form a foam that fills the head space. Leaving the bottle at rest, one can witness the slow evolution of the foam as it drains and the bubbles become smaller in number but larger in size, until the foam eventually disappears (in about $0.5 \mathrm{~h}$ ).

Foam is one of the signature characteristics of beer and is due to the interaction of its various components-most notably proteins from malt and acids from hop [23,24]. However, when ingredients or processing are not adequate, foaming can get out of control. Extensive research has gone into determining the causes of gushing. There is agreement that primary gushing is caused by a specific kind of - particularly hydrophobic - proteins named hydrophobins, which are secreted by various species of fungi that can infect malt $[3,25,26]$. However, there is not yet a reliable way to predict and prevent this. Of course, the main way of prevention is to avoid using infected ingredients, but that might not always be possible when large amounts of beer are produced or in years that crop infection is high. Besides necessarily dealing with bubble nucleation, growth, and motion, research in this topic is heavily oriented towards the complex chemistry of beer, looking to disentangle the interactions that lay behind primary gushing in order to find effective prevention mechanisms. From the physics point of view, an appropriate approach is to look for a way to dissolve all bubbles that might be stabilized by surface-active molecules. In this respect, treatment with ultrasonic pressure waves might offer interesting possibilities [27].

\section{Buoyancy-Driven Flows in Beer}

As was explained in Section 2, buoyancy-driven flows can be extremely effective in enhancing gas-liquid mass transfer in carbonated beverages. Even down to the level of a single bubble, buoyancy-induced convection is able to significantly speed up bubble growth. Enríquez et al. [28] performed experiments in which $\mathrm{CO}_{2}$ bubbles were grown under very well-controlled supersaturation conditions. Although during some time the bubble radius was observed to scale with the square root of the time, as predicted by Epstein and Plesset [8], after a while this growth rate became much faster due to the effect of buoyancy-induced convection. In fact, as the bubble grows, it depletes the $\mathrm{CO}_{2}$ in its vicinity. Since the density of water increases with the concentration of dissolved $\mathrm{CO}_{2}$, the liquid that surrounds the bubble becomes lighter and rises, giving birth to a plume which takes away the 
depleted liquid and by mass conservation brings water rich in $\mathrm{CO}_{2}$ closer to the bubble, which in turn enhances mass transfer and makes bubbles grow faster.

Convection also plays a very important role in the degassing of beer once it is poured into a glass. When beer is exposed to ambient air, Henry's law dictates that the concentration of $\mathrm{CO}_{2}$ at the liquid's surface must be very small, since the proportion of this gas in air is likewise small (let us ignore for a moment the existence of a foam head). Consequently, diffusion will establish a flux of $\mathrm{CO}_{2}$ from the bulk liquid to the free surface, from where this gas will be lost to the ambient environment. At a first glance, though, this degassing mechanism seems to be very inefficient, due to the small value of the diffusivity of $\mathrm{CO}_{2}$ in water (around $D \approx 2 \times 10^{-9} \mathrm{~m}^{2} / \mathrm{s}$ ). Indeed, the typical time that diffusion alone would take to deplete a glass of height $h=10 \mathrm{~cm}$ would be $t_{\text {degass }} \sim h^{2} / D=5 \times 10^{6} \mathrm{~s}$, or around 57 days. This estimation is in strong contrast with our daily-life experience, that suggests that a beer glass left unattended on a table loses the gas in a mater of tens of minutes. A tempting explanation is that the bubbles that naturally form at the glass surface drain $\mathrm{CO}_{2}$ from deep inside the glass and transport it to the free surface in its rising motion. However, experiments in Champagne reveal that this is not the case, and that indeed this mechanism only accounts for about $20 \%$ of the gas loss, being the other $80 \%$ due to the diffusive flux of gas to the surface [1]. Actually, both mechanisms couple together to achieve the fast degassing of the liquid that we have all experienced at some point. As bubbles rise to the free surface, they drag some liquid with them that, when it reaches the surface, goes back to the bottom by mass conservation. This induces a recirculatory motion inside the glass that transports the $\mathrm{CO}_{2}$ along with the liquid to the free surface, where diffusion is strong enough to transfer the gas to the atmosphere. Moreover, in a similar fashion as occurs in beer tapping, this recirculating flow also promotes the faster growth of the bubbles from nucleation sites at the walls. Besides its obvious contribution to the taste and texture of beer, another important mission of the foam head is to create a barrier that precludes the diffusion of carbon dioxide to the ambient environment.

To conclude this section, we would like to mention a curious phenomenon that is very much connected to the convective motion induced by rising bubbles that we have just described; namely, that of waves in stout beers [29]. These waves become apparent when a pint of this beer is poured and consist of periodic spatial oscillations in the concentration of bubbles that propagate downwards along the wall of the glass (see Figure 2 and Video S3 in the Supplemental Materials). This is a very remarkable feature, as the main motion of the bubbles in the glass is naturally upwards. Robinson et al. [29] developed a mathematical model of this flow, which they associated with a shear instability between the bubbly liquid that rises in the central portion of the glass and the downwards movement that occurs near the walls. Benilov et al. [30] further contributed to explain the cause of this recirculatory motion. Although it seems logical to think that bubbles close to the center of the pint rise faster than those near the glass due to the friction exerted by the wall, they concluded that the shape of the container is essential to the shape of the recirculation pattern.

Interestingly, the mathematical model of Robinson et al. [29] points out the similarity between these waves and others found in natural and industrial flows. Firstly, waves in stout beers are analogous to the roll waves that occur in a thin liquid film, such as the long waves that appear in water sliding downhill in a rainy day. And secondly, similar waves have been detected by remote sensing in oil extraction boreholes, although they have not been seen directly. 


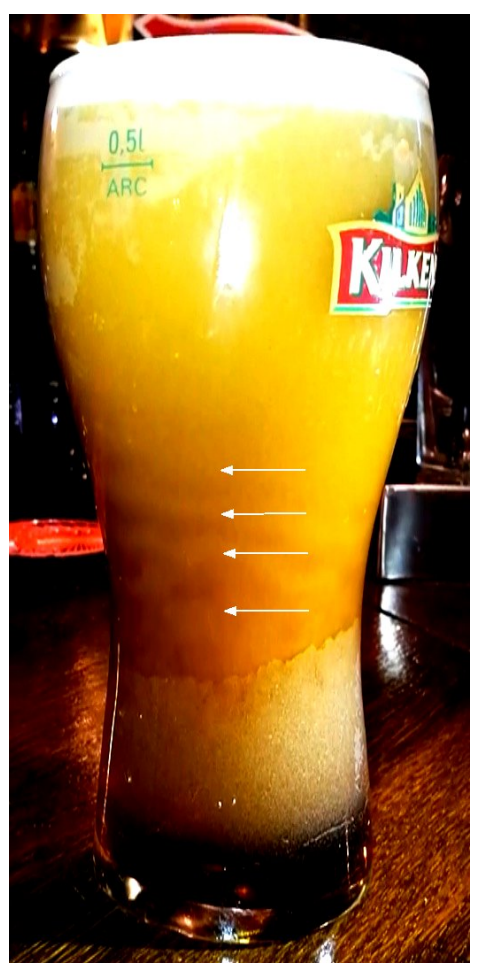

Figure 2. Waves in a freshly served pint of a stout beer. The arrows mark the location of the peaks in bubble concentration. The downward motion of the waves can be observed in the associated Video S3 (see Supplemental Materials). Note also the chaotic oscillation of the front that separates the recirculation bubble trapped by the thickening of the glass near the bottom and the downwards flow of foam near the wall.

\section{Bubble Growth with Multiple Gas Species: Stout Beers}

Stout beers owe much of their characteristic taste-especially the creamy texture of their head-to the fact that they contain a mixture of dissolved carbon dioxide and nitrogen. Including dissolved nitrogen in a carbonated beer has two effects that make these beers special: on one hand it reduces the acidity, and on the other makes bubbles smaller. Nonetheless, it has a downside, which is that the low solubility of nitrogen makes the time needed for bubbles to grow (and rise) much larger than for $\mathrm{CO}_{2}$ ones; therefore, the need to patiently wait for one's stout pint. Furthermore, while drafting a beer from a tap generates enough mixing to promote bubble growth, opening a can or bottle does not, which makes necessary an additional help to generate the foam.

The reason for this slower mass transfer was explored by Lee et al. [31], who extended the model of Liger-Belair et al. [32] for the growth of bubbles in Champagne (where they are composed solely of $\mathrm{CO}_{2}$ ) to the case of a mixture between nitrogen and carbon dioxide. In few words, and without entering into the details of the mathematical model, the amount of $\mathrm{CO}_{2}$ contained in a stout beer is smaller than the saturation amount at ambient pressure, as the gas mixture used to pressurize the beer contains mostly nitrogen. For instance, [31] report partial bottling pressures of $P_{\mathrm{CO}_{2}}=0.80$ and $P_{N_{2}}=3.00$ bars for $\mathrm{CO}_{2}$ and nitrogen, respectively. Thus, when the beer is exposed to ambient pressure, it is still weakly oversaturated in carbon dioxide, which then makes nitrogen the main gas that will make bubbles grow from nucleation sites. Nonetheless, once the bubble is formed, some $\mathrm{CO}_{2}$ will flow into it until the partial pressure of this gas matches the saturation pressure $P_{\mathrm{CO}_{2}}$. Consequently, because nitrogen is much slower in making bubbles grow due to its low solubility (about 50 times less soluble than $\mathrm{CO}_{2}$ ), the process of bubble growth becomes equally slow in a stout beer compared to a carbonated beer. 
As we said above, the mixing generated when a beer is draft from a tap is still able to make nitrogen bubbles grow reasonably fast, but this is not the case when these beers are poured from a can or bottle. To promote bubble growth, these containers have in their interior some devices, or widgets, that generate a turbulent gas jet when the bottle or can depressurizes (i.e., when it is open). For instance, Guinness cans (see Figure 3) use a plastic sphere pressurized with nitrogen at the bottling pressure that breaks when exposed to ambient pressure. This widget enhances the formation of bubbles when pouring a beer into a glass, quickly forming the creamy head.

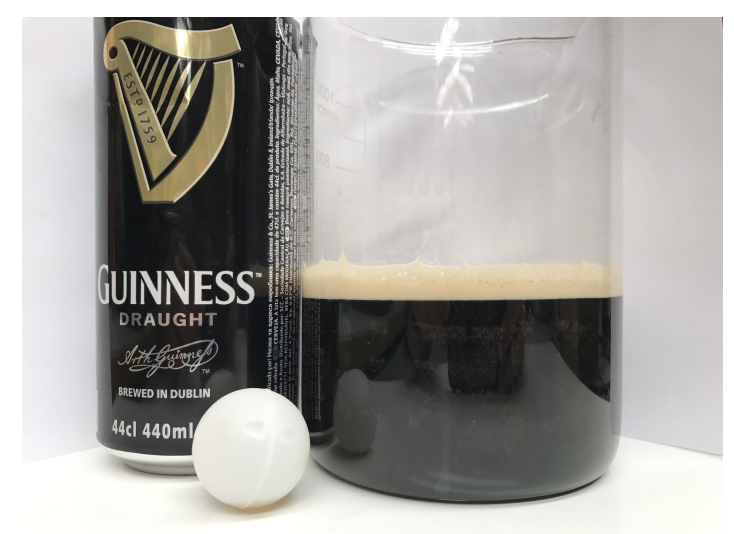

Figure 3. Widget inside a Guinness can. The hollow ball is filled up with pressurized gas at the same pressure than the can. When the can is opened, the widget breaks and generates a turbulent gas jet that introduces the mixing necessary to trigger foam formation.

A point which deserves special attention is the mechanism proposed by Liger-Belair et al. [32] to explain bubble growth in Champagne, and that is of application to beers (stout or not). As a result of the manipulation of the bottles as well as the manufacturing of the beverage (specially crafted beers), cellulose fibers exist naturally inside beer containers. These fibers are hollow, with inner diameters of a few microns. The small size of these fibers makes capillary confinement effective to retain micrometric gas pockets in their inside. This gas pocket can be formed by capillary rise when the fiber is in touch with gas during the bottling process. Figure 4 shows one of these gas pockets trapped inside cellulose fiber from cotton immersed in beer.

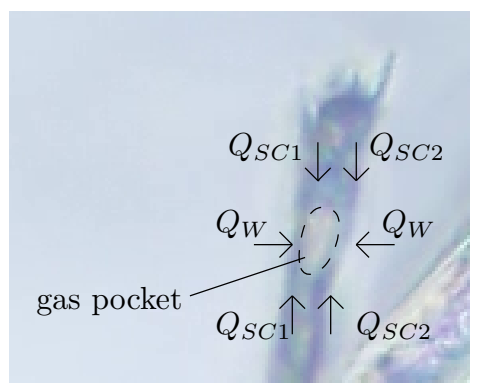

Figure 4. Microscope image of a gas pocket inside a cellulose fiber immersed in beer. The arrows describe the different fluxes of gases ( 1 for $\mathrm{CO}_{2}$ and 2 for $\mathrm{N}_{2}$ that make the bubble grow. $Q_{S C}$ and $Q_{W}$ stand for diffusion flux across the spherical cap and the fiber's wall, respectively.

Bubbles then grow from these nucleation sites by diffusion. Then, when the gas pocket achieves a critical size and reaches the fiber's mouth, a tiny bubble comes out, but a small portion of the gas remains inside the fiber and shrinks back to the initial position, therefore allowing the process to start over. This formation process can be divided into two stages: the slow growth of the gas pocket, and the fast detachment of the bubble. The growth rate of the gas pocket depends on the diffusion fluxes of 
dissolved gases in the liquid though the spherical caps $\left(Q_{S C}\right)$ at the ends of the gas pocket and the walls $\left(Q_{W}\right)$ of the cellulose fiber (Figure 4$)$, which are porous to the gases. This process is illustrated in Figure 5 and in the associated movie S2 in the supplemental material.

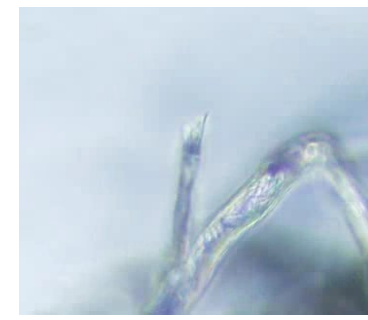

(a)

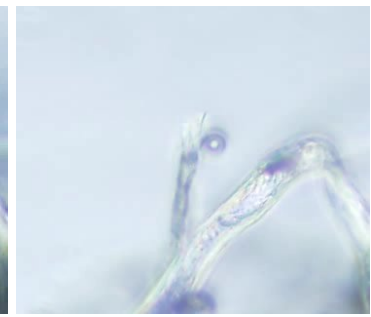

(b)

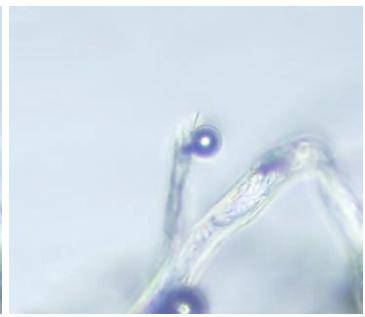

(c)

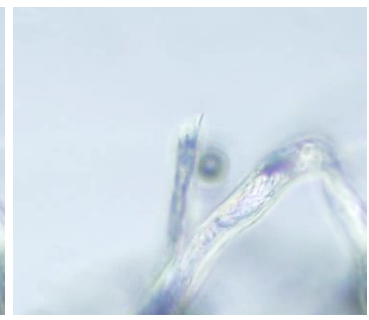

(d)

Figure 5. Microscope images, acquired by the authors, of bubble nucleation from a cellulose fiber at (a) $t=0 \mathrm{~ms}$, (b) $t=300 \mathrm{~ms}$, (c) $t=400 \mathrm{~ms}$, and (d) $t=600 \mathrm{~ms}$. The fiber is immersed in a petri dish containing freshly opened pale lager beer (Mahou Clásica), which has a bottling pressure of 5 bars $(\zeta=4)$. See video S2.

\section{Conclusions}

In the previous sections we have described a selection of physical phenomena involving bubbles that can be commonly observed in beer. Some of these phenomena are quite intriguing, as they defy intuition -at least in a first thought. For instance, we have described bubbly waves that sink instead of rising, how an apparently mild impact on a beer bottle is able to trigger a beer volcano, or how shaking reduces the pressure inside a container instead of increasing it.

Most, if not all, of these phenomena have important implications for the beer industry, as they affect the way the beverage is brewed or transported. However, the focus of the article has been put on describing these processes from a qualitative yet rigorous point of view, with the aim of luring beer fans into becoming more interested in the Physics behind this beverage. A remarkable feature is that once the physics behind the phenomenon is understood, very similar processes are observed to occur in Nature, in applications apparently unconnected to a beer glass.

Thus, our beer glass—-besides being physically pleasant—can be seen as a small-scale laboratory of a very rich variety of physical phenomena that range from geological flows to water waves rolling downhill on a rainy day.

Supplementary Materials: The following are available online at www.mdpi.com/2306-5710/3/3/38/s1. Video S1: Sudden explosion of foam after impacting a champagne bottle. Video S2: Bubble growing from a gas pocket confined inside a cellulose fiber. Video S3: Foam waves running downwards near the wall of the glass of a stout beer. Video S4: Comparison of the evolution of two clouds of $\mathrm{CO}_{2}$ bubbles, one with gravity and another one without.

Acknowledgments: We acknowledge the support of the Spanish Ministry of Economy and Competitiveness through grants DPI2014-59292-C3-1-P and DPI2015-71901-REDT, partly funded through European Funds. We are also grateful to Jaume Lluis Tartera, from the beer company Mahou-San Miguel, for pointing out to the authors some very interesting aspects of the role of foam in beer.

Author Contributions: All authors participated equally in writing this review.

Conflicts of Interest: The authors declare no conflict of interest.

\section{References}

1. Liger-Belair, G. The Physics and Chemistry behind the Bubbling Properties of Champagne and Sparkling Wines: A State-of-the-Art Review. J. Agric. Food Chem. 2005, 53, 2788-2802.

2. Denny, M. Froth! The Science of Beer; Johns Hopkins University Press: Baltimore, MD, USA, 2009.

3. Bamforth, C.; Russell, I.; Stewart, G. Beer: A Quality Perspective; Academic Press: Oxford, UK, 2011.

4. Smith, F.L.; Harvey, A.H. Avoid common pitfalls when using Henry's law. Chem. Eng. Prog. 2007, 103, 33-39. 
5. Jones, S.; Evans, G.; Galvin, K. Bubble nucleation from gas cavities-A review. Adv. Colloid Interface Sci. 1999, 80, 27-50.

6. De Gennes, P.G.; Brochard-Wyart, F.; Quéré, D. Capillarity and Wetting Phenomena: Drops, Bubbles, Pearls, Waves; Springer Science \& Business Media: New York, NY, USA, 2013.

7. Bamforth, $\mathrm{C}$. The relative significance of physics and chemistry for beer foam excellence: Theory and practice. J. Inst. Brew. 2004, 110, 259-266.

8. Epstein, P.; Plesset, M. Stability of gas bubbles in liquid-gas solutions. J. Chem. Phys. 1950, 18, 1505-1509.

9. Liger-Belair, G.; Religieux, J.B.; Fohanno, S.; Vialatte, M.A.; Jeandet, P.; Polidori, G. Visualization of mixing flow phenomena in champagne glasses under various glass-shape and engravement conditions. J. Agric. Food Chem. 2007, 55, 882-888.

10. Liger-Belair, G.; Cilindre, C.; Beaumont, F.; Jeandet, P.; Polidori, G. Evidence for ascending bubble driven flow patterns in champagne glasses, and their impact on gaseous $\mathrm{CO}_{2}$ and ethanol release under standard tasting conditions. Bubble Sci. Eng. Technol. 2012, 4, 35-48.

11. Beaumont, F.; Liger-Belair, G.; Bailly, Y.; Polidori, G. A synchronized particle image velocimetry and infrared thermography technique applied to convective mass transfer in champagne glasses. Exp. Fluids 2016, 57, 1-7.

12. Beaumont, F.; Liger-Belair, G.; Polidori, G. Unveiling self-organized two-dimensional (2D) convective cells in champagne glasses. J. Food Eng. 2016, 188, 58-65.

13. Munasinghe, P.C.; Khanal, S.K. Biomass-derived syngas fermentation into biofuels: Opportunities and challenges. Bioresour. Technol. 2010, 101, 5013-5022.

14. Rodríguez-Rodríguez, J.; Casado-Chacón, A.; Fuster, D. Physics of beer tapping. Phys. Rev. Lett. 2014, 113, 214501.

15. Mantic-Lugo, V.; Cayron, A.; Brun, P.T.; Gallaire, F. Beer tapping: Dynamics of bubbles after impact. J. Phys. Conf. Ser. 2015, 656, 012029.

16. Griffiths, R.W. Entrainment and stirring in viscous plumes. Phys. Fluids A. 1991, 3, 1233.

17. Vega-Martínez, P.; Rodríguez-Rodríguez, J.; van der Meer, D.; Sperl, M. Drop tower setup to study the diffusion-driven growth of a foam ball in supersaturated liquids under microgravity conditions. Microgravity Sci. Technol. 2017, in press.

18. Zhang, Y.; Kling, G. Dynamics of Lake Eruptions and Possible Ocean Eruptions. Annu. Rev. Earth Planet. Sci. 2006, 34, 293-324.

19. Rogers, M.; Morris, S. Buoyant plumes and vortex rings in an autocatalytic chemical reaction. Phys. Rev. Lett. 2005, 95, 0245505.

20. Mott, R.; Woods, A. A model of overturn of $\mathrm{CO}_{2}$ laden lakes triggered by bottom mixing. J. Volcan. Geotherm. Res. 2010, 192, 151-158.

21. Vreme, A.; Pouligny, B.; Nadal, F.; Liger-Belair, G. Does shaking increase the pressure inside a bottle of champagne? J. Colloid Interface Sci. 2015, 439, 42-53.

22. Jones, S.F.; Evans, G.M.; Galvin, K.P. The cycle of bubble production from a gas cavity in a supersaturated solution. Adv. Colloid Interface Sci. 1999, 80, 51-84.

23. Asano, K.; Hashimoto, N. Isolation and characterization of foaming proteins of beer. J. Am. Soc. Brew. Chem. 1980, 38, 129-137.

24. Bamforth, C. The foaming properties of beer. J. Inst. Brew. 1985, 91, 370-383.

25. Stübner, M.; Lutterschmid, G.; Vogel, R.F.; Niessen, L. Heterologous expression of the hydrophobin FcHyd5p from Fusarium culmorum in Pichia pastoris and evaluation of its surface activity and contribution to gushing of carbonated beverages. Int. J. Food Microbiol. 2010, 141, 110-115.

26. Shokribousjein, Z.; Deckers, S.M.; Gebruers, K.; Lorgouilloux, Y.; Baggerman, G.; Verachtert, H.; Delcour, J.A.; Etienne, P.; Rock, J.M.; Michiels, C.; et al. Hydrophobins, beer foaming and gushing. Cerevisia 2011, $35,85-101$.

27. Sahu, K.K.; Hazama, Y.; Ishihara, K.N. Gushing in canned beer: The effect of ultrasonic vibration. J. Colloid Interface Sci. 2006, 302, 356-362.

28. Enríquez, O.; Sun, C.; Lohse, D.; Prosperetti, A.; van der Meer, D. The quasi-static growth of $\mathrm{CO}_{2}$ bubbles. J. Fluid Mech. 2014, 741, R1.

29. Robinson, M.; Fowler, A.; Alexander, A.; O’Brien, S. Waves in Guinness. Phys. Fluids 2008, $20,067101$.

30. Benilov, E.; Cummins, C.; Lee, W. Why do bubbles in Guinness sink? Am. J. Phys. 2012, 81, 88. 
31. Lee, W.; McKechnie, J.S.; Devereux, M.G. Bubble nucleation in stout beers. Phys. Rev. E 2011, 83, 051609.

32. Liger-Belair, G.; Voison, C.; Jeandet, P. Modeling non-classical heterogeneous bubble nucleation from cellulose fibers: Application to bubbling in carbonated beverages. J. Phys. Chem. B 2005, 109, 14573-14580. 